EPJ Web of Conferences 106, 03008 (2016)

DOI: $10.1051 /$ epjconf/201610603008

(C) Owned by the authors, published by EDP Sciences, 2016

\title{
Application of the Subgroup Decomposition Method (SDM) for Reactor Simulation
}

\author{
Nathan Roskoff, William Walters, and Alireza Haghighat \\ Nuclear Engineering Program, Mechanical Engineering Department, Virginia Tech, Arlington, \\ VA, USA
}

\begin{abstract}
The performance of the TITAN-SDM algorithm for solving a reactor pressure vessel dosimetry problem is evaluated. Douglass and Rahnema recently developed the he subgroup decomposition method (SDM); a methodology which directly couples a consistent coarse-group transport calculation with a set of "decomposition sweeps" to provide a fine-group flux spectrum. The SDM has been implemented into the TITAN threedimensional transport code and has been shown to accurately solve core criticality problems while significantly reducing computation time. This paper addresses the use of SDM for fixed-source problems. The VENUS-2 dosimetry benchmark problem is selected with an emphasis on fast neutron analysis; therefore, material cross sections are generated from the BUGLE-96 library considering neutron energies greater than $0.1 \mathrm{MeV}$. The accuracy and efficiency of TITAN-SDM is evaluated by comparison with a standard TITAN multigroup calculation.
\end{abstract}

\section{Introduction}

Recently, Douglass and Rhanema developed a novel method for performing multigroup transport calculations for core physics application, i.e. core criticality calculation. This method, referred to as Subgroup Decomposition Method (SDM) [1], is an extension of the standard cross-section condensation process [2] that preserves spectral accuracy as well as the energy-angle coupling which is generally lost in standard condensed-group transport calculations. The SDM can effectively generate a detailed finegroup flux spectrum from a coarse-group transport calculation.

The SDM algorithm is implemented into the TITAN three-dimensional $\mathrm{S}_{\mathrm{N}}$ transport code [3], referred to as TITAN-SDM. TITAN-SDM has been previously demonstrated for core criticality calculations on both a one- and two-dimensional Very High Temperature Reactor (VHTR) core model [4]. The results demonstrated that, for a core criticality problem, the SDM algorithm is capable of significantly reducing computation time while maintaining adequate accuracy in both the eigenvalue and flux distribution.

In this paper, the performance of TITAN-SDM for a fixed-source reactor dosimetry problem is evaluated based on a model of the VENUS-2 benchmark problem [5]. Material cross-section information is generated from the BUGLE-96 library [6] which includes 47-group neutron and 20-group gamma data derived from ENDF/B-VI. The analysis considers fast neutron dosimetry; neutrons with energy of

This is an Open Access article distributed under the terms of the Creative Commons Attribution License 2.0, which permits unrestricted use, distribution, and reproduction in any medium, provided the original work is properly cited. 
$0.1 \mathrm{MeV}$ or greater are of concern, therefore a 26-group cross-section library is used. Reaction rates of 4 different dosimeters $\left({ }^{58} \mathrm{Ni}(n, p),{ }^{103} \mathrm{Rh}\left(n, n^{\prime}\right),{ }^{237} \mathrm{~Np}(n, f)\right.$, and $\left.{ }^{27} \mathrm{Al}(n, \alpha)\right)$ located at 34 positions are calculated. Accuracy of the TITAN-SDM algorithm is evaluated by comparing these calculations with those from a standard multigroup TITAN calculation.

This paper is organized as follows: Sect. 2 introduces the basic theory of the SDM methodology, Sect. 3 briefly discusses the implementation of the SDM into the TITAN 3-D transport code, Sect. 4 describes the VENUS-2 benchmark problem and provides results from a reference 26-group TITAN calculation, Sect. 5 presents an evaluation of the performance of the TITAN-SDM, and Sect. 6 summarizes the results.

\section{Theory}

As elaborated in Ref. [1], the important aspect of the SDM formulation is accounting for the energyangle coupling effect of the total group section which is typically neglected in standard multigroup formulation [7].

Therefore, the SDM utilizes an alternative form of the multigroup transport equation known as the consistent multigroup formulation which accounts for this energy-angle coupling effect by the introduction of a multigroup correction term [2]. Equation (1) presents a "corrected" multigroup transport equation,

$$
\hat{\Omega} \cdot \nabla \psi_{g}(\boldsymbol{r}, \hat{\Omega})+\sigma_{g} \psi_{g}(\boldsymbol{r}, \hat{\Omega})=S_{g}(r, \hat{\Omega})+\delta_{g}(\boldsymbol{r}, \hat{\Omega}),
$$

where $g$ refers to the fine-group index, $\psi_{g}(\boldsymbol{r}, \hat{\Omega})$ is the angular flux at position $\boldsymbol{r}$ and direction $\hat{\Omega}, \sigma_{g}$ is the total macroscopic cross-section, $S_{g}$ is the total multigroup source, and $\delta_{g}$ is the multigroup correction term. The correction term $\delta_{g}$ is given by Eq. (2).

$$
\delta_{g}(\boldsymbol{r}, \hat{\Omega})=\int_{g} d E\left(\sigma_{g}(\boldsymbol{r})-\sigma_{g}(\boldsymbol{r}, \boldsymbol{E})\right) \psi(\boldsymbol{r}, \hat{\Omega}, E) .
$$

The above formulation provides the difference in group reaction rate, the "standard" group crosssection [7] versus the angular-dependent group cross-section. Interested readers should consult Refs. [1] and [8].

\section{SDM Implementation into TITAN}

The implementation of the SDM algorithm into a 3-D deterministic transport code is a four-step process. These steps are as follows: (i) a fine-group flux distribution is used to collapse the fine-group cross sections into a coarse-group library; (ii) coarse-group transport calculations are performed; (iii) finegroup fluxes are obtained through a recondensation sweep; and (iv) a fine-group flux-update scheme is performed. Each step will be briefly discussed in the following sections. An interested reader should consult Reference 4 for a detailed derivation of the necessary equations.

\subsection{Coarse-group Cross-section Library Development}

The coarse-group cross sections, denoted by subscript $h$, are defined as an average of the fine-group cross sections, denoted by subscript $g$, contained within the coarse-group, or subgroups. Equation (3) shows a general equation to calculate a type $x$ coarse-group cross section, $\sigma_{x, h}$.

$$
\sigma_{x, h}(\boldsymbol{r})=\frac{\sum_{g \in h}\left[\sigma_{x, g}(\boldsymbol{r}) \phi_{g}(\boldsymbol{r})\right]}{\sum_{g \in h} \phi_{g}(\boldsymbol{r})} .
$$

Where $\sigma_{x, g}$ is the fine-group type $x$ cross-section and $\phi_{g}$ is fine-group scalar flux. 


\section{$15^{\text {th }}$ ISRD}

\subsection{Coarse-group Transport}

Equation (4) presents the coarse-group transport equation.

$\hat{\Omega} \cdot \nabla \psi_{h}(\boldsymbol{r}, \hat{\Omega})+\sigma_{h}(\boldsymbol{r}) \psi_{h}(\boldsymbol{r}, \hat{\Omega})=\sum_{h^{\prime}=1}^{H} \int_{4 \pi} d \Omega^{\prime} \sigma_{s, h^{\prime} \rightarrow h}\left(\boldsymbol{r}, \hat{\Omega}^{\prime} \cdot \hat{\Omega}\right) \psi_{h^{\prime}}\left(\boldsymbol{r}, \hat{\Omega}^{\prime}\right)+\delta_{h}(\boldsymbol{r}, \hat{\Omega})+q_{h}(\boldsymbol{r}, \hat{\Omega})$.

Where $\psi_{h}$ is the coarse-group angular flux, $\sigma_{h}$ is the total coarse-group cross section, $\sigma_{s, h^{\prime} \rightarrow h}$ is the coarse-group differential scattering cross-section, $\delta_{h}$ is the multigroup correction term, and $q_{h}$ is the coarse-group independent source, which is defined as

$$
q_{h}(\boldsymbol{r}, \hat{\Omega})=\sum_{g \in h} q_{g}(\boldsymbol{r}, \hat{\Omega}),
$$

where $q_{g}$ is the fine-group independent source.

\subsection{Fine-group Decomposition}

Once the coarse-group fluxes and flux moments are known, it is desired to obtain fine-group fluxes. This is accomplished by solving a one-pass, fine-group transport equation,

$$
\hat{\Omega} \cdot \nabla \psi_{g}(\boldsymbol{r}, \hat{\Omega})+\sigma_{g}(\boldsymbol{r}) \psi_{g}(\boldsymbol{r}, \hat{\Omega})=\sum_{h^{\prime}=1}^{H} \int_{4 \pi} d \Omega^{\prime} R_{s, h^{\prime} \rightarrow g}\left(\boldsymbol{r}, \hat{\Omega}^{\prime} \cdot \hat{\Omega}\right) \psi_{h^{\prime}}\left(\boldsymbol{r}, \hat{\Omega}^{\prime}\right)+q_{g}(\boldsymbol{r}, \hat{\Omega}),
$$

where

$$
R_{s, h^{\prime} \rightarrow g}(\boldsymbol{r})=\frac{\sum_{g^{\prime} \epsilon h^{\prime}} \sigma_{s, g^{\prime} \rightarrow g}(r) \phi_{g^{\prime}}(r)}{\sum_{g^{\prime} \in h^{\prime}} \phi_{g^{\prime}}(r)} .
$$

The $R$ constant is referred to as the "subgroup decomposition cross-section". It represents the scattering source in subgroup $g$ from coarse-group $h^{\prime}$. Therefore, if coarse-group fluxes are known, they provide a way to decompose the coarse-group source into the respective fine groups.

\subsection{Flux-update Scheme}

This step ensures the convergence of the fine-group flux; it should be noted that this step can be excluded if the coarse-group tolerances are set tight enough, though the flux-update scheme increases solution accuracy for a give number of iterations and also decreases computation time [9]. This step is simply a fine-group transport sweep,

$$
\hat{\Omega} \cdot \nabla \psi_{g}(\boldsymbol{r}, \hat{\Omega})+\sigma_{g}(\boldsymbol{r}) \psi_{g}(\boldsymbol{r}, \hat{\Omega})=\sum_{g^{\prime}=1}^{G} \int_{4 \pi} d \boldsymbol{\Omega}^{\prime} \sigma_{s, g^{\prime} \rightarrow g}\left(\boldsymbol{r}, \hat{\Omega}^{\prime} \cdot \hat{\Omega}\right) \psi_{g^{\prime}}\left(\boldsymbol{r}, \hat{\Omega}^{\prime}\right)+q_{g}(\boldsymbol{r}, \hat{\Omega}) .
$$

\section{VENUS-2 Model Description and Reference Solution Results}

The VENUS-2 benchmark problem [5] has been issued by OECD/NEA with the objective to test current computational methods for calculating neutron flux in MOX-fuel critical experiments. The measured data for this benchmark are equivalent fission fluxes and reaction rates obtained using ${ }^{58} \mathrm{Ni}(n, p)$, ${ }^{115} \mathrm{In}\left(n, n^{\prime}\right),{ }^{103} \mathrm{Rh}\left(n, n^{\prime}\right),{ }^{64} \mathrm{Zn}(n, p),{ }^{237} \mathrm{~Np}(n, f)$, and ${ }^{27} \mathrm{Al}(n, \alpha)$ detectors positioned at 34 locations on the core midplane of the reactor. These detectors are located at the core center, in the outer core region, the core baffle, the water reflector, the core barrel, and neutron pad. 




\begin{tabular}{cc}
\hline Dosimeter ID & Location \\
\hline 1,2 & Inner baffle \\
$3-9$ & Outer baffle \\
$10-17$ & Barrel \\
18,19 & Neutron pad \\
20 & Central hole \\
$21-29$ & Water gap \\
$30-34$ & Reflector \\
\hline
\end{tabular}

Figure 1. Meshing and material distribution used in the core level of VENUS2 TITAN model. Dosimeter locations are identified by (ם).

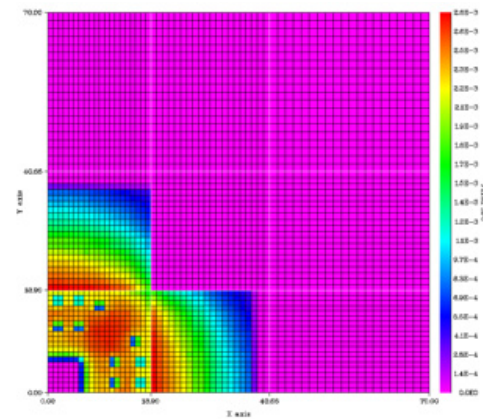

a. Source distribution, core axial-level



b. 26-group source spectrum

Figure 2. VENUS-2 fixed-source specification.

\subsection{TITAN Model}

We have developed a 3-D model of the VENUS reactor using TITAN. The model consists of 8 axiallevels and a total of 17,775 fine meshes. Figure 1 shows the discretized model of the core level along with the 34 dosimeter locations (all of the dosimeters are located on the core midplane) with a numerical identification number for reference purposes. The reactor core is characterized by three homogenous regions: (1) an inner region with $\mathrm{UO}_{2} 3.0$ w/o enriched, (2) an outer region composed of $\mathrm{UO}_{2} 4.0$ w/o enriched, and (3) a MOX region with 2.0 w/o $\mathrm{UO}_{2}$ and 2.7 w/o Pu. Fast neutrons are of primary concern in this problem, therefore we will only consider neutron energies of $0.1 \mathrm{MeV}$ or greater. The 26-group multigroup cross sections used are derived from the BUGLE-96 cross section library [6]. The 3-D fixed-source distribution used was generated from the data provided in the benchmark specification. Figure 2a shows the source distribution at the core midplane and Fig. 2b source spectrum.

\subsection{Reference Solution Results}

The 26-group reference solution is calculated using an $\mathrm{S}_{8}$ quadrature set (a total of 80 directions), $\mathrm{P}_{1}$ scattering order, and flux convergence of $10^{-3}$. Figure 3 shows normalized, by the maximum flux 


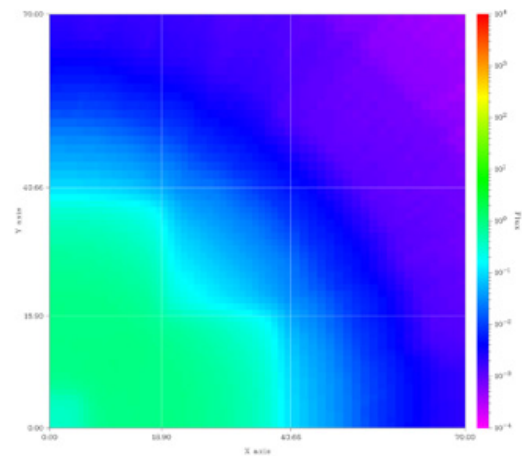

a. Group 1, 14.2-19.6 MeV

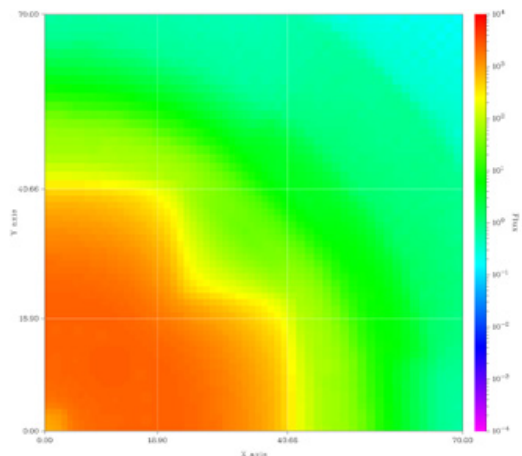

b. Group 18, 1.00-1.35 MeV

Figure 3. 26-group reference solution flux distributions; normalized by the maximum flux in group 1.

Table 1. The upper energies of the first 26 groups of BUGLE-96.

\begin{tabular}{cc|cc|cc|cc|cc}
\hline & $\begin{array}{c}\text { Upper E } \\
{[\mathbf{M e V}]}\end{array}$ & $\mathbf{g}$ & $\begin{array}{c}\text { Upper E } \\
{[\mathbf{M e V}]}\end{array}$ & $\mathbf{g}$ & $\begin{array}{c}\text { Upper E } \\
{[\mathbf{M e V}]}\end{array}$ & $\begin{array}{c}\text { Upper E } \\
\mathbf{[ M e V}]\end{array}$ & $\begin{array}{c}\text { Upper E } \\
\mathbf{g} \mathbf{M e V}]\end{array}$ \\
\hline 1 & 19.60 & 7 & 6.07 & 13 & 2.37 & 19 & 1.00 & 25 & 0.30 \\
2 & 14.20 & 8 & 4.97 & 14 & 2.35 & 20 & 0.82 & 26 & 0.18 \\
3 & 12.20 & 9 & 3.68 & 15 & 2.23 & 21 & 0.74 & & \\
4 & 10.00 & 10 & 3.01 & 16 & 1.92 & 22 & 0.61 & & \\
5 & 8.61 & 11 & 2.73 & 17 & 1.65 & 23 & 0.50 & & \\
6 & 7.41 & 12 & 2.47 & 18 & 1.35 & 24 & 0.37 & & \\
\hline
\end{tabular}

in group 1, reference solution flux spectrum for groups 1 and 18; since the source spectrum peaks at energy group 18 the neutron flux in the core also peaks in this group, between 1.00 and $1.35 \mathrm{MeV}$.

The model was run on a single processor allocated with $8 \mathrm{~GB}$ of memory; the TITAN calculated reference solution required $\sim 110$ seconds of computation time.

\section{Testing TITAN-SDM}

TITAN-SDM is tested with different coarse-group structures and the effects of algorithm control parameters are investigated. Table 1 presents the upper energy bounds of the 26-group structure of BUGLE-96.

Table 2 presents the different coarse-group structures that are tested as identified by the fine-group number that defines the boundary of the coarse-group. The $2 \mathrm{cg}$ and $3 \mathrm{cg}$ structures were selected based on the energy structure of the BUGLE-96 library. The $4 \mathrm{cg}, 6 \mathrm{cg}, 8 \mathrm{cg}$, and $10 \mathrm{cg}$ structures were selected by observing trends of the 26-group material cross sections; in areas of strong cross-section gradients more coarse groups were considered. New algorithm control parameters were introduced to check the convergence of coarse-group cross-section and coarse-group flux tolerance.

First, an analysis of the solution accuracy is presented through dosimeter reaction rate ratio, $r$, as compared to the standard multigroup TITAN. This ratio is given as

$$
r=\frac{\left(R_{D}\right)_{T I T A N-S D M}}{\left(R_{D}\right)_{\text {Ref. }}},
$$

where

$$
R_{D}=\sum_{g=1}^{G} \sigma_{g, D} \phi_{g}
$$


Table 2. Coarse-group testing structures.

\begin{tabular}{|c|c|c|c|c|c|c|c|c|c|c|}
\hline \multirow{3}{*}{$\begin{array}{l}\text { Test } \\
\text { Case }\end{array}$} & \multicolumn{10}{|c|}{ Coarse-group ID \# } \\
\hline & 1 & 2 & 3 & 4 & 5 & 6 & 7 & 8 & 9 & 10 \\
\hline & \multicolumn{10}{|c|}{ Fine-group ID \# (lower energy bound) } \\
\hline $1 \mathrm{cg}$ & 26 & & & & & & & & & \\
\hline $2 \mathrm{cg}$ & 18 & 26 & & & & & & & & \\
\hline $3 \mathrm{cg}$ & 6 & 18 & 26 & & & & & & & \\
\hline $6 \mathrm{cg}$ & 6 & 18 & 20 & 22 & 24 & 26 & & & & \\
\hline $10 \mathrm{cg}$ & 6 & 18 & 19 & 20 & 21 & 22 & 23 & 24 & 25 & 26 \\
\hline
\end{tabular}

Table 3. Analysis of the effect of coarse-group structures on solution accuracy and timing.

\begin{tabular}{|c|c|c|c|c|c|c|c|c|c|}
\hline \multirow[b]{2}{*}{ Case } & \multicolumn{2}{|c|}{${ }^{58} \mathrm{Ni}$} & \multicolumn{2}{|c|}{${ }^{103} \mathbf{R h}$} & \multicolumn{2}{|c|}{${ }^{237} \mathrm{~Np}$} & \multicolumn{2}{|c|}{${ }^{27} \mathrm{Al}$} & \multirow{2}{*}{$\begin{array}{c}\text { Time } \\
{[\mathrm{s}]}\end{array}$} \\
\hline & $\mathbf{R M S}^{1}$ & $\mathbf{M A X}^{2}$ & RMS & MAX & RMS & MAX & RMS & MAX & \\
\hline $1 \mathrm{cg}$ & 0.9999 & 0.9994 & 0.9998 & 0.9993 & 0.9999 & 0.9990 & 0.9999 & 0.9992 & 658.8 \\
\hline $2 \mathrm{cg}$ & 0.9994 & 0.9984 & 0.9992 & 0.9978 & 0.9993 & 0.9980 & 0.9997 & 0.9990 & 578.7 \\
\hline $3 \mathrm{cg}$ & 0.9997 & 0.9989 & 0.9997 & 0.9992 & 0.9998 & 0.9990 & 1.0000 & 0.9992 & 650.7 \\
\hline $10 \mathrm{cg}$ & 0.9996 & 0.9984 & 0.9991 & 0.9958 & 0.9994 & 0.9973 & 1.0000 & 0.9992 & 738.7 \\
\hline
\end{tabular}

Table 4. Analysis of the effect of coarse-group flux tolerances on solution accuracy and timing for a 3 coarse-group structure.

\begin{tabular}{cccccccccc}
\hline Flux Tol. & \multicolumn{2}{c}{} & \multicolumn{2}{c}{${ }^{\mathbf{5 8}} \mathbf{N i}$} & \multicolumn{2}{c}{${ }^{103} \mathbf{R h}$} & \multicolumn{2}{c}{${ }^{237} \mathbf{N p}$} & \multicolumn{2}{c}{${ }^{27} \mathbf{A l}$} & \multirow{2}{*}{ Time [s] } \\
\cline { 2 - 9 } & $\mathbf{R M S}^{1}$ & $\mathbf{M A X}$ & RMS & MAX & RMS & MAX & RMS & MAX & \\
\hline $10^{-3}$ & 0.9997 & 0.9989 & 0.9997 & 0.9992 & 0.9998 & 0.9990 & 1.0000 & 0.9992 & 650.7 \\
$10^{-2}$ & 0.9997 & 0.9989 & 0.9996 & 0.9980 & 0.9997 & 0.9988 & 1.0000 & 1.0007 & 620.5 \\
$10^{-1}$ & 1.0008 & 1.0017 & 0.9980 & 0.9812 & 1.0002 & 0.9946 & 1.0002 & 1.0020 & 627.9 \\
\hline
\end{tabular}

${ }^{1}$ Root-mean-squared value of dosimeter ratio, ${ }^{2}$ maximum dosimeter ratio.

$R_{D}$ is the detector response, $\sigma_{g, D}$ is the group $g$ detector of type $D$ cross-section, and $\phi_{g}$ is the fine-group scalar flux.

Reaction rates are calculated at each of the 34 positions, Fig. 1, for the following 4 dosimeter reaction types: ${ }^{58} \mathrm{Ni}(n, p),{ }^{103} \mathrm{Rh}\left(n, n^{\prime}\right),{ }^{237} \mathrm{~Np}(n, f)$, and ${ }^{27} \mathrm{Al}(n, \alpha)$. Maximum absolute dosimeter ratio discrepancy and root-mean-squared (RMS) values are examined for each reaction type. Then, a detailed break down of times required for the different steps of the algorithm is discussed to identify areas for possible improvement.

\subsection{Accuracy Analysis}

Table 3 shows results of testing the different coarse-group structures, see Table 1, on the solution accuracy as well as computation time. For each coarse group, the maximum detector reaction rate ratio and root-mean-squared value are presented along with computation time. The fine-group flux is converged to $10^{-1}$ and the coarse-group fluxes have either converged to $10^{-3}$ or have met the maximum number of iterations allowed per subgroup iteration; this value is set to 10 iterations for all cases.

Table 3 demonstrates that number of coarse-group groups as well as selection of group boundaries has some impact on the accuracy of the calculation and the amount of computation time required. The fastest computation time is the $2 \mathrm{cg}$ case. The results indicate that the $3 \mathrm{cg}$ test case is the most accurate, showing the smallest $r$ values.

The impact of coarse-group flux tolerance is evaluated on the $3 \mathrm{cg}$ test case. Table 4 presents the effects of the coarse-group flux tolerances on solution accuracy and computation time. 


\section{$15^{\text {th }}$ ISRD}

Table 5. Total number of iterations for multigroups and SDM transport calculations.

\begin{tabular}{cc}
\hline $\begin{array}{c}\text { Reference Multigroup } \\
\text { (26 groups) }\end{array}$ & $\begin{array}{c}\text { SDM } \\
\text { (3 coarse groups) }\end{array}$ \\
\hline 324 & $134 *$ \\
\hline${ }^{*}$ Comprised of 122 coarse-group and 12 fine-group calculations.
\end{tabular}

Table 6. Fractional computation times ${ }^{1}$ required for different steps of the TTIAN-SDM algorithm.

\begin{tabular}{cccc}
\hline $\begin{array}{c}\text { Coarse-group } \\
\text { Transport }\end{array}$ & \multicolumn{2}{c}{$\begin{array}{c}\text { Fine-group Related } \\
\text { Calculations }\end{array}$} \\
\hline Total & Source & Total & Source \\
\hline 0.27 & 0.22 & 0.73 & 0.60 \\
\hline${ }^{1}$ Total subgroup iteration time is 85.3 & seconds.
\end{tabular}

Table 4 indicates that, as expected, using a tighter coarse-group flux tolerance will produce more accurate reaction rates at the expense of computation time. As the flux tolerance is decreased, the accuracy of dosimeter reaction rates also decreases; the computation time also decreases slightly.

It should be noted that the required computation time for these TITAN-SDM calculations require significantly more, by nearly five times, computation time than the standard multigroup approach.

\subsection{Analysis of Timing}

Table 5 presents a comparison of the total number of transport calculations that are completed in the reference multigroup calculation and SDM algorithm. The SDM calculation total is broken down into the number of coarse-group transport calculations and fine-group transport calculations.

Table 5 demonstrates that the TITAN-SDM transport calculation requires significantly fewer, by a factor of $\sim 2.5$, number of iterations than the standard multigroup calculation. So, the question remains: why is the measured computation time significantly larger for TITAN-SDM? To answer this question, we examine the amount of computation time spent in different segment of the TITAN-SDM.

The total time required for a single subgroup (SG) loop is comprised of the sum of each of the four steps, previously discussed in Sect. 3: (1) coarse-group (CG) transport, (2) fine-group (FG) decomposition, (3) fine-group flux-update, and (4) coarse-group cross-section generation (the time required for this step is negligible compared to the other steps). To simplify this discussion we will consider the time spent on coarse-group transport, Step 1, and the combined time spent on fine-group related calculations, Steps 2 and 3. Table 6 presents the times required for the different steps of a single subgroup iteration.

Table 6 demonstrates that $\sim 70 \%$ of the total subgroup iteration time is spent in on fine-group related calculations, while the coarse-group transport only accounts for $\sim 30 \%$ of the iteration time. This analysis shows that a significant amount, approximately $70 \%$, of the computational costs of the SDM algorithm, for this fixed source problem, are spent obtaining the detailed flux spectrum and not on the coarse-group transport. It is concluded that a major portion, nearly $80 \%$, of the computation time in both the coarse-group transport and fine-group related calculations is spent calculating the source terms.

\subsection{Discussion}

It has been demonstrated that TITAN-SDM requires significantly more time than the standard multigroup calculation for solving this fixed-source problem. While, the number of transport iterations for the TITAN-SDM is significantly lower, a significant speedup was observed when solving the criticality problem. To address this, Table 7 presents the total number of coarse-group transport 


\section{EPJ Web of Conferences}

Table 7. Number of coarse-group iterations per subgroup iteration.

\begin{tabular}{cc}
\hline Criticality & Fixed-source \\
\hline 600 & 30 \\
\hline
\end{tabular}

calculations per subgroup iteration for the 1-D HTTR calculation [4] and the fixed-source problem presented here.

Table 7 shows that there are significantly more, nearly 20 times, coarse-group calculations per subgroup iteration for the criticality problem than there are for the fixed-source calculation. This analysis implies that the fixed-source calculation presented here is not computationally efficient because not enough coarse-group calculations are completed within a subgroup iteration. Hence, it is essential to explore development of more efficient calculations of determination a fine-group source using the coarse-group results.

\section{Conclusions}

In this paper we have tested the TITAN-SDM algorithm for fixed-source shielding application on the VENUS-2 benchmark problem. The SDM algorithm is shown to accurately calculate dosimeter responses. TITAN-SDM is sensitive to the number of coarse-groups selected and the definition of the coarse-group boundaries. It has been shown that in spite of significant reduction of number of transport sweeps, currently TITAN-SDM requires significantly more computation time than standard multigroup transport; the majority, $\sim 60 \%$, of this time is spent on source calculation for the finegroup decomposition loop. The speedups observed in the criticality calculations are not observed for this fixed-source problem. We believe that this finding can be attributed to the small number of coarsegroup transport calculations for a fixed-source calculation. Efforts are underway to explore alternative approaches for reducing the computation time used for source calculations.

The authors thank Drs. Saam Yasseri and Ce Yi and Profs. Farzad Rahnema and Glenn Sjoden of the Georgia Institute of Technology for their comments and suggestions.

\section{References}

[1] S. Douglass, F. Rahnema, Annals of Nuclear Energy 48, 84 (2012)

[2] S. Douglass, F. Rahnema, Annals of Nuclear Energy 40, 200 (2012)

[3] C. Yi, A. Haghighat, Nuc. Sci. Eng. 164, 3 (2010)

[4] N. Roskoff, W. Walters, A. Haghighat, C. Yi, G. Sjoden, PHYSOR (2014), accepted

[5] C.Y. Han, C. Shin, H.C. Kim, J.K. Kim,N. Messaoudi, B.C. Na, NEA/NSC/DOC(2004)

[6] E. White, Oak Ridge National Laboratory, D00185-ALLCP-00 (1996)

[7] G. Bell, S. Glasstone, Nuclear Reactor Theory (Krieger Publishing Company, 1970)

[8] S. Douglass, Ph.D. thesis, Georgia Institute of Technology (2012)

[9] S. Yasseri, F. Rahnema, Annals of Nuclear Energy 60, 235 (2013) 